\title{
Management and Attitudes of Patients Victims Accidental Exposure to Blood at the Day Care Unit of the Central Hospital of Yaoundé (Cameroon) From January 2007 to December 2016
}

\section{Defo Defo ${ }^{1}$, Emmanuel Armand Kouotou, 2,3*, Ulrich Nguena Feungue ${ }^{4}$, Joseline Domo ${ }^{1}$, Antonin Wilson Ndjitoyap Ndam ${ }^{2,5}$, Dahlia Noelle Tounouga ${ }^{1}$ and Elie Claude Ndjitoyap Ndam ${ }^{2}$}

${ }^{1}$ Day Care Unit, Central Hospital of Yaoundé, Cameroon

${ }^{2}$ Department of Internal Medicine and Specialties, Faculty of Medicine and Biomedical Sciences, University of Yaoundé I, Yaoundé, Cameroon

${ }^{3}$ Yaoundé University Teaching Hospital, Yaoundé, Cameroon

${ }^{4}$ University Hospital of Treichville, Abidjan, Ivory Coast

${ }^{5}$ General Hospital of Yaoundé, Cameroon

*Corresponding author: Pr Emmanuel Armand Kouotou, Dermatologist, Venereologist, Allergologist, Department of Internal Medicine and Specialties, Faculty of Medicine and Biomedical Sciences, University of Yaoundé I, Yaoundé, Cameroon, P.O. Box: 8314 Yaoundé, Cameroon, Tel: +237-696-95-50-83; +237-679-84-43-60

\begin{abstract}
Background: There is little data on Accidental Exposure to Blood (AEB) in Cameroon.

Objectives: to learn about the management of AEB among professional and non-professional patient at the day care unit of the Central Hospital of Yaoundé.

Method: This was a 10-year retrospective study in which data concerning socio-demographic characteristics, exposure and consultation circumstances, measures taken after the accident, the status of the source patient and of the patient consultant and the prescribed ARV protocol were analyzed.

Results: Six hundred files were selected, including $49.2 \%$ professional exposure and $50.2 \%$ non-professional. The mean age was $30 \pm 9.7$ years with extremes of 11 and 67 years. The main circumstances of $A E B$ were needle stick (professional AEB) and rape (non-professional AEB). Prophylaxis with triple antiretroviral therapy has been prescribed to all patients.
\end{abstract}

Conclusion: Non-professional and professional AEB are found in equivalent proportions in our series. Triple antiretroviral therapy was prescribed for all patients. Staff awareness policies could be developed to respect universal precautionary measures.

\section{Keywords}

Accidental exposure to blood, Professional, Nonprofessional, Hand washing, Triple antiretroviral therapy, Yaoundé (Cameroon)

\section{List of Abbreviations}

AEB: Accidental exposure to blood; Duovir-Aluvia: Duovir - Lopinavir/r; TDF-3TC-Aluvia: Ténofovir - Lamuvidine - Lopinavir/r; AZT-3TC-EFV: Zidovudine - Lamuvidine - Efavirenz; AZT-3TC-IDV: Zidovudine - Lamuvidine Indinavir; AZT-3TC-ATV/r: Zidovudine - Lamuvidine Atazanavir/r

\section{Introduction}

Accidental exposure to blood (AEB) is defined as contact with contaminated blood or body fluids, with a needle prick, cutting with a sharp object, or contact

Citation: Defo D, Kouotou EA, Feungue UN, Domo J, Ndam AWN, et al. (2021) Management and Attitudes of Patients Victims Accidental Exposure to Blood at the Day Care Unit of the Central Hospital of Yaoundé (Cameroon) From January 2007 to December 2016. Int J Virol AIDS 8:077. doi. org/10.23937/2469-567X/1510077

Accepted: October 09, 2021: Published: October 11, 2021

Copyright: (C) 2021 Defo D, et al. This is an open-access article distributed under the terms of the Creative Commons Attribution License, which permits unrestricted use, distribution, and reproduction in any medium, provided the original author and source are credited. 
with contaminated blood or fluid on a wound, nonintact skin or mucous membrane [1]. AEB constitutes one of the modes of transmission of HIV.

These accidents mainly concern caregivers, people who handle medical waste or those who have been sexually assaulted [1,2]. According to studies, the average risk of HIV infection after percutaneous exposure is $0.32 \%$ [3]. Non-professional AEB are poorly documented but can constitute up to $56 \%$ of $A E B$ according to certain studies [2].

After an AEB, immediate measures should be taken such as cleaning the wound and assessing the risk. The risk assessment includes among others: time between exposure and consultation, the severity of the exposure, the nature of the biological fluid responsible, the serological and clinical status of the source person [3]. In addition, the decision to initiate antiretroviral (ARV) prophylaxis must be made as soon as possible within 4 hours of exposure with a maximum delay of 48 hours [4]. The post-exposure prophylaxis currently recommended is triple antiretroviral therapy [4]. The other recommended measures are cleaning with soap and water or bleach diluted to the tenth on the skin and abundant rinsing with physiological saline in the mucous membranes [3].

In Cameroon, a study conducted in 2013 at the Central Hospital of Yaoundé by Nouetchognou, et al. showed that $36.7 \%$ of healthcare giver had experienced at least one episode of AEB in the last 3 months of exercise [5]. Mbock, et al. in 2015 found a generally poor level of knowledge, attitudes and practices of paramedical staff vis-à-vis the AEB [6].

In our context there are very few data on the management of non-professional AEB. The aim of our study was to study the management and attitudes of patients who are victims of non-professional $A E B$ and to update the data on professional $A E B$ consulting at the day care unit of the Central Hospital of Yaoundé.

\section{Material and Methods}

\section{Study design, setting and participants}

It was a retrospective study conducted from January 2007 to December 2016 at the day care unit of the Central Hospital of Yaoundé (CHY). It is a structure specializing in screening and management of people living with HIV (PLWHIV). The active file of PLWHIV followed in this health facility is around 40,000 patients and constitutes the largest cohort in Cameroon. This day care unit has a dermatology department and an electronic archiving system.

We included in the study any patient, health staff or not spontaneously coming to consult for AEB and whose file was usable. The sampling was consecutive and not exhaustive.
We have considered as professional $A E B$ any $A E B$ affecting health personnel when carrying out more or less invasive care involving different biological fluids and also cleaner exposed to the bites of injectable material left in the bins in hospitals. We have considered as unprofessional $A E B$ any $A E B$ primarily related to unprotected, unsafe sex and other circumstances outside the hospital.

\section{Study procedure}

A survey form has been established in order to consolidate the data recorded in the $A E B$ register of the day care unit. This sheet notified: Demographic characteristics (age, sex, profession), date of consultation, date of exposure, duration of exposure, nature of exposure, measures taken after the accident, patient status source, the status of the consulting patient, the decision made, the ARV protocol prescribed.

\section{Data analysis}

We encoded and analyzed the data using Epi info software version 3.5.3 and designed the graphs and figures using the Excel 2010 office software. The Chi2 test allowed us to determine associations between our qualitative variables. The statistical significance threshold was set at $5 \%$.

\section{Ethical considerations}

This study was granted an ethical clearance by the Ethical Review Board of the Faculty of Medicine and Biomedical Sciences, University of Yaoundé I, Cameroon. Additionally, administrative authorizations were obtained from the directory of the Yaoundé Central Hospital before the beginning of the study. The anonymity was respected, and the data collected in the files was only used in the context of this study.

\section{Results}

During this study period, 611 patients were victims of $A E B$ among which 11 files were unusable. A total of 600 files complied with our selection criteria and were selected.

\section{Sociodemographic characteristics:}

The population of patients consulting for AEB was essentially female $(426 / 600 ; 71 \%)$ with a sex ratio $\mathrm{M} / \mathrm{F}$ of 0.4 . The mean age was $30 \pm 9.7$ years with extremes of 11 and 67 years. The most affected age group was $25-$ 29 years of age (Table 1 ).

Less than 24 hours after the AEB 400 patients (66.7\%) consulted while $11 \%$ consulted after the scheduled time for post-AEB prophylaxis meaning more than $48 \mathrm{~h}$ later (Table 2).

This study allowed us to note a predominance of non-professional AEB (305/600; 50.8\%). In the general population, rape was by far the most common cause 
Table 1: Distribution of patients by age and sex.

\begin{tabular}{|l|ll|}
\hline \multirow{2}{*}{ Age $(\mathbf{N}=\mathbf{6 0 0})$} & Males & Females \\
\hline < 15 years & $\mathrm{n}(\%)$ & $\mathrm{n}(\%)$ \\
$\mathbf{1 5 - 1 9}$ years & $2(0.6)$ & $16(3.8)$ \\
$\mathbf{2 0 - 2 4}$ years & $26(14.9)$ & $93(11.3)$ \\
$\mathbf{2 5 - 2 9}$ years & $55(31.6)$ & $93(21.8)$ \\
$\mathbf{3 0 - 3 4}$ years & $36(20.7)$ & $66(15.5)$ \\
$\mathbf{3 5 - 3 9}$ years & $20(11.5)$ & $46(10.8)$ \\
$\mathbf{4 0 - 4 4}$ years & $12(6.9)$ & $24(5.6)$ \\
$\mathbf{7} \mathbf{4 5}$ years & $22(12.6)$ & $40(9.4)$ \\
Total & $174(100)$ & $426(100)$ \\
\hline
\end{tabular}

Table 2: Distribution of patients according to the time between exposure and consultation.

\begin{tabular}{|lll|}
\hline Duration (in hours) & Number of case (N) & Percentage (\%) \\
\hline $\mathbf{2} \mathbf{2 4}$ & $\mathbf{4 0 0}$ & $\mathbf{6 6 . 7}$ \\
{$[\mathbf{2 4 - 4 8 ]}$} & 134 & 22.3 \\
$\mathbf{2} \mathbf{4 8}$ & 66 & 11 \\
Total & 600 & 100 \\
\hline
\end{tabular}

of AEB (220/305; 72.1\%), while among nursing staff, the main cause of $A E B$ was the needlestick of needles (141/295; 47.8\%).

After $A E B$, the first attitude is the disinfection of the site with running water $(280 / 600 ; 46.7 \%)$ followed by the usage of bleach $(116 / 600 ; 19.3 \%)$. In addition, $133 / 600(23.1 \%)$ took no action.

Table 3 illustrates the nature of the exposure and the various measures taken by patients to avoid possible HIV infection.

Forty-four percent (264/600) of source patient was HIV positive and the serological status was unknown in $50.2 \%$ (301/600) patient (Table 4).

In our study 94.2\% (565/600) received post-exposure prophylaxis made of triple antiretroviral therapy comprising either 2 Reverse Transcriptase Inhibitors + 1 Protease Inhibitor or 2 Reverse Transcriptase Inhibitors +1 Non-nucleoside inhibitor reverse transcriptase (Figure 1).

At the day care unit of the Central Hospital of

Table 3: Distribution of patients by type of exposure and measures taken by patients.

\begin{tabular}{|c|c|c|}
\hline & $\mathbf{N}$ & Percentage (\%) \\
\hline A. Nature of exposure $(\mathrm{N}=600)$ & & \\
\hline Personnel health care (professional AEB) & 295 & 100 \\
\hline Pitting (needlestick) & 141 & 47.8 \\
\hline Cutting (bistouri) & 7 & 2.4 \\
\hline Spalsch (biological fluid) & 29 & 9.8 \\
\hline Not precised & 118 & 40 \\
\hline General population (non professional AEB) & 305 & 50.8 \\
\hline rape & 220 & 72.2 \\
\hline Condom break & 46 & 15.1 \\
\hline Unprotected sex & 31 & 10.2 \\
\hline Bite & 8 & 2.6 \\
\hline B. Measures taken by patients $(\mathrm{N}=600)$ & & \\
\hline Disinfection (with running water and soap) & 280 & 46.7 \\
\hline Bleach & 116 & 19.3 \\
\hline Gynecological consultation & 28 & 4.7 \\
\hline Alcohol & 12 & 2 \\
\hline Pression & 10 & 1.7 \\
\hline Pill (levonorgestrel) & 8 & 1.3 \\
\hline Chlorhexidine & 4 & 0.7 \\
\hline Sodium Hypochlorite & 3 & 0.5 \\
\hline Povidone-lodine & 3 & 0.5 \\
\hline Cotrimoxazole & 1 & 0.2 \\
\hline Doxycycline & 1 & 0.2 \\
\hline Disinfection with saline & 1 & 0.2 \\
\hline None & 133 & 22.1 \\
\hline Total & 600 & 100 \\
\hline
\end{tabular}


Table 4: Distribution of patients according to the serological status of the source patient and the consulting patient.

\begin{tabular}{|l|l|l|l|}
\hline Serology & HIV+ & HIV- & Not done/unknown \\
& N (\%) & N (\%) & N (\%) \\
\hline Source person & $264(44)$ & $35(5.8)$ & $301(50.2)$ \\
Consulting patient $^{* *}$ & $14(2.3)$ & $571(95.2)$ & $15(2.5)$ \\
\hline
\end{tabular}

*Source person: Person from whom the potentially contaminated biological product (professional AEB) comes or from whom the non-professional AEB comes; ${ }^{* *}$ Consulting patient: Person victim of AEB.

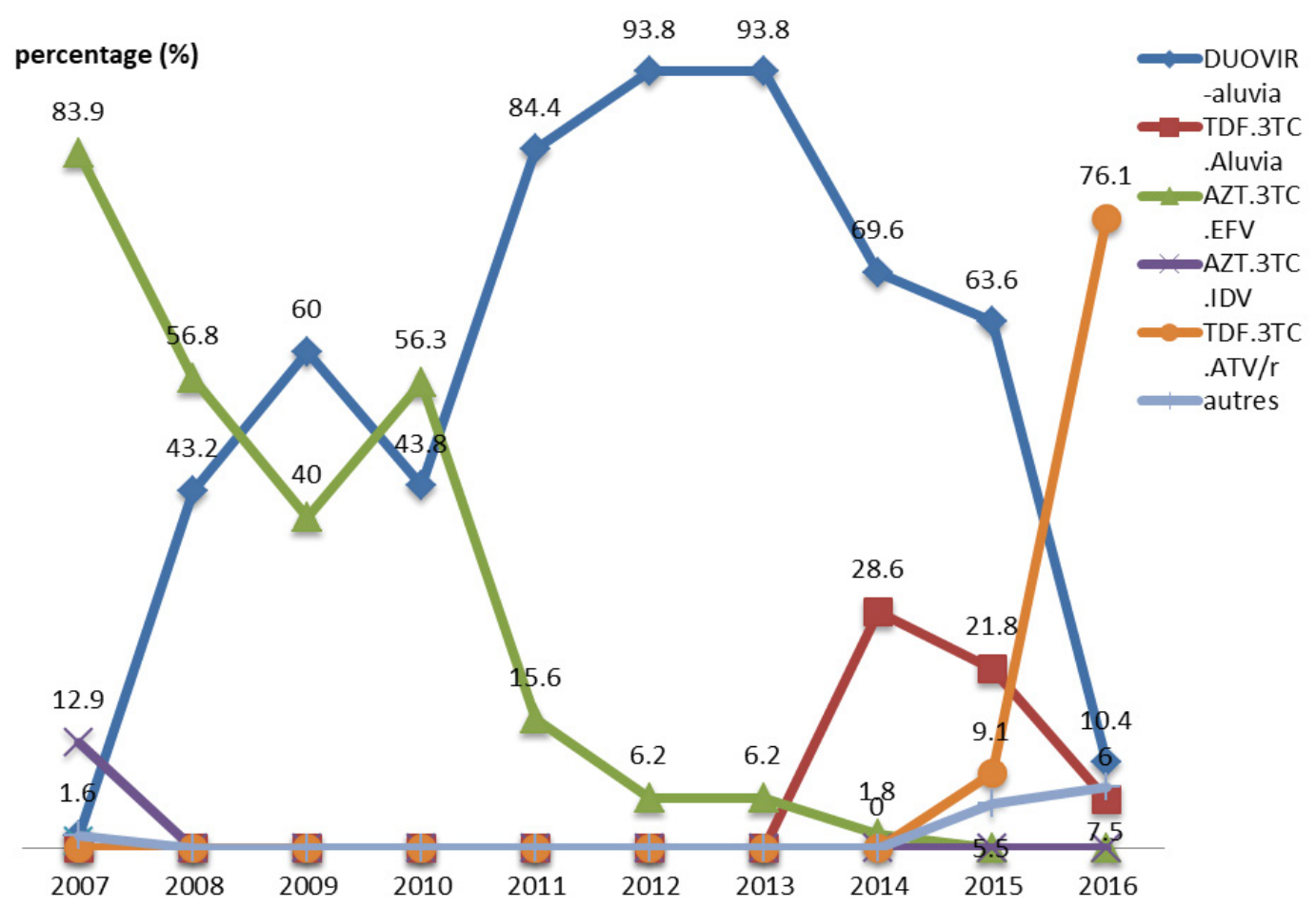

Figure 1: Distribution of patients according to the ARV protocol used and by year.

Yaoundé the most used ARV protocol in 2007 was AZT3TC-LPV/ r (DUOVIR-ALUVIA); from 2013 zidovudine (AZT) was replaced by tenofovir disoproxil fumarate (TDF), (Figure 1).

\section{Discussion}

This study allowed us to note the proportions of professional and non-professional $A E B$ among all the patients consulting for $A E B$ in the day care unit, to describe the different circumstances of $A E B$, to describe the attitudes of patients towards $A E B$ and to specify the therapeutic options for hospital care over the years.

Over a 10-year period, we identified 600 female victims of AEB (426/600; 71\%) with a sex ratio of 0.4 . This predominance of women has been noted by other African authors [7-9]. This could be explained by a high proportion of women among the medical staff on the one hand and the higher frequency of rapes among women than among men on the other hand [10]. However, Ehui, et al. in his Ivorian series of 2007 noted a male predominance (sex ratio $M / F$ of 1.4 ) among the victims of AEB [11]. This difference could be linked to a fluctuation in sampling since 3 years later, the same author finds rather a female predominance (sex-ratio $\mathrm{M}$ / F of 0.6) as in most series of the literature [10].

The average age in our series was $30 \pm 9.7$ years with extremes of 11 and 67 years. These results are comparable to those of Ehui, et al. in Ivory Coast and Gounongbé, et al. in Benin who respectively noted the average ages of $33.8 \pm 7.4$ years [11] and $35.7 \pm 8.8$ years [8] in their studies.

The recommended consultation period for initiating post-exposure prophylaxis is 48 hours $[3,4]$. In our series $89 \%$ of individuals consulted within 48 hours of the onset of AEB. The Tunisian, Ivorian and French studies report proportions of consultations within less than 48 hours respectively of $89.8 \%$ [9], 93.7\% [10] and 93\% [1]. Most patients who suffered from AEB in our study, as in these Tunisian, Ivorian and French series, consulted within less than 48 hours; this demonstrates patient awareness and interest in reducing the risk of post-exposure infection. However, the proportion of our consulting patients less than 24 hours after exposure in our study was $66.7 \%$. This proportion is lower than that the one of Hajjaji, et al. who noted a proportion of $81.3 \%$ [9]. This difference could be due to the difference in our study 
populations; our study covered all the patients received for AEB including personnel health care and the general population while that of Hajjaji, et al. was made up of trainee doctors; a homogeneous study population with a priori a level of medical knowledge superior to ours.

Among $A E B$ in the health personnel, injections by syringe were the most represented $47.8 \%$ (141/295). Needlestick injuries remain the leading cause of professional AEB worldwide. This proportion of professional AEB due to needle sticks was $83 \%$ in 2007 in Ivory Coast [11], 85\% in Tunisia in 2010 [9], 68\% in Togo in 2011 [12]. Practices such as recapping needles, dragging needles in garbage bags could explain this high frequency of needlestick bites [11]. This would also require the establishment of communication programs for behavior change at national or even international level. As for non-professional AEB, rape was at the top of the line with a frequency of $36.7 \%$ compared to all AEB. Our results are similar to those of Rouveix, et al. in France which reports a prevalence of AEB by sexual exposure of $31 \%$ [2]. In our series, as far as non-professional AEB is concerned, rape constitutes $72.1 \%$ (220/305), condom breaks $15.1 \%(46 / 305)$ and unprotected sex $10.2 \%$ (31/305). These data can be superimposed on those of Ehui, et al. in 2010 which found $57.8 \%(74 / 128)$ of rapes, $22.6 \%(29 / 128)$ of condom breakage and $16.4 \%(21 / 128)$ of unprotected sex regarding non-professionals AEB in Abidjan [10].

Cleaning with soap (46.7\%) and disinfection with bleach (19.3\%) were the main measures taken by our patients after an AEB. Zougrana, et al. in Burkina Faso in 2014 noted a similar frequency $(30 / 64 ; 46.8 \%)$ of people victims of AEB who cleaned the site of exposure with water and soap [13]. Contrarily to Moroccan series where the frequency of this gesture was 68\% [14]. In addition, in this same Moroccan series, almost all participants (97\%) did hand disinfection after AEB, and in $83 \%$ of cases the disinfectant was with bleach [14]. Among our participants, disinfection concerned $86.5 \%$ of cases and was done with bleach in $26.5 \%$ of cases. This disparity could be explained by a poor level of mastery of precautionary measures among our participants compared to that of Asseray, et al. who rather had a high level of control over precautionary measures [14]. Indeed, our study population was heterogeneous and included both the medical staff and the general population, while the Moroccan study consisted only of medical staff.

In our study, $44 \%$ of our source patients were HIV positive. Our results are comparable to those found in several African works. Indeed for [11] in Ivory Coast and [15] in Algeria these proportions were $46.7 \%$ and $51.7 \%$ respectively. In contrast, Gounongbé, et al. in Benin had found a much lower proportion (10.2\%). During our work, the patients consulted were mixed and almost proportional $(49.2 \%$ of health personnel and $50.2 \%$ of the general population); due to the presence of half of the source patients in the hospital, performing the test in these patients was easier. Gounongbé, et al. in Benin noted a high proportion of non-professional exposure (80\%); and the serology of the majority of these non-professional AEB source patients was unknown probably because these non-professional $A E B$ source patients were not in a hospital setting [8]. This situation must have reduced the proportion of source HIV positive patients $(10.2 \%)$ in their study. Almost all of the consultant patients (97.5\%) in our study had their HIV serology. Darouiche, et al. reported that all patients with BSE have been tested in their series. This high rate of screening of source patients is favored by the accessibility or even the free testing for HIV testing set up by the health policies of the different states [16].

The prescription of post-exposure prophylaxis is variable in the literature and depends on the risk of contamination which can be minimal, low or high [3]. If the consultation is done within 48 hours, the prescription of a prophylaxis can be discussed depending on the level of risk and the type of exposure. Thus, in our study where we generally had a high risk of contamination increased by a high proportion of AEB through sexual penetration (297/600; 49.5\%); the frequency of post-exposure prophylaxis was $94.2 \%$ $(565 / 600)$. In the cohort of children from Monpoux, et al. in France only $18.8 \%$ (6/48 AEB by sexual penetration, 3 by other means of AEB estimated to be high risk) received post-exposure prophylaxis [1]. The post-exposure prophylaxis prescribed for our patients was triple antiretroviral therapy. It is the most recommended and currently prescribed protocol, as shown by several studies $[2,10,13]$. Other therapies, in particular monotherapy with zidovudine and dual therapies combining zidovudine, remain alternatives [3]. Indeed, zidovudine is the first molecule whose efficacy has been proven in prophylaxis after exposure to HIV, in particular during the CDC control case study in 1995 , with a notable reduction in seroconversion by $80 \%$ [3]. However, due to the development of resistance to zidovudine, it should no longer be prescribed alone [3]. Overall, new prophylaxis protocols recommend the association of two nucleoside reverse transcriptase inhibitors and one protease inhibitor.

\section{Limits of the study}

The main difficulties encountered during our study were the absence in certain files of some information such as profession, precision of AEB circumstances. Almost all the files had no follow-up information; which did not allow us to have information on compliance and the proportion of post-exposure seroconversion.

\section{Conclusion}

Non-professional and professional AEB are found in equivalent proportions in our series. Needlestick and 
rape are the two main circumstances of $A E B$. The most common action taken by patients after an $A E B$ in our series is hand washing. The triple antiretroviral therapy recommended today has been prescribed for our patients. Staff awareness policies could be developed to respect universal precautionary measures and reflection should be made on the fight against violence.

\section{Declarations}

\section{Availability of data and material}

Data will be made available on request addressed to the corresponding author.

\section{Conflict of interest}

The authors declare that they have no conflicts of interest with regard to this article.

\section{Funding}

This research did not receive any specific grant from funding agencies in the public, commercial, or not-forprofit sectors.

\section{Authors' contributions}

EAK and DD conceived and designed the study. JD and DD collected the data. EAK, and UNF analyzed and interpreted the data. EAK and UNF drafted the manuscript. EAK, DD, UNF, JD, AWNN and ECNN reviewed and revised the manuscript. All authors read and approved the final version of the manuscript.

\section{Acknowledgments}

The authors are very grateful to the director of the Central Hospital of Yaoundé where our study took place and to all of the staff of the Day Care Unit for their kind assistance during data collection.

\section{References}

1. Monpoux F, Berlioz M, Pradier C, Haas H, Boutte P (2002) HIV exposure accidents in children. Experience at the emergency admission services of the $\mathrm{CHU}$ of Nice. Arch Pediatr 9: 232-237.

2. Rouveix E, Bouvet E, Vernat F, Chansombat M, Hamet G, et al. (2014) Management of accidental exposure to HIV: The COREVIH 2011 activity report. Médecine et Maladies Infectieuses 44: 112-126.

3. Raffenne L, Bodard L, Meudec A (2005) Accidents d'exposition au sang : Conduite à tenir. EMC - Médecine 2: 291-299.

4. Sornicle G, Pereira G, Guéry A, Landre C, Boulain T (2009) Accidents d'exposition au sang en réanimation et lors des procédures de circulation extracorporelle. Réanimation 18: 459-465.
5. Nouetchognou JS, Ateudjieu J, Jemea B, Mbanya D (2016) Accidental exposures to blood and body fluids among health care workers in a Referral Hospital of Cameroon. BMC Res Notes 9: 94.

6. Mbock HC (2015) Accidents d'exposition au sang à l'Hôpital Central de Yaoundé: Connaissances, attitudes et pratiques du personnel paramédical. Health Sciences and Diseases.

7. Sheth SP, Leuva AC, Mannari JG (2016) Post Exposure prophylaxis for occupational exposures to HIV and Hepatitis $\mathrm{B}$ : Our Experience of thirteen years at a rural based tertiary care teaching hospital of western India. J Clin Diagn Res 10: OC39-OC44.

8. Gounongbé DFCA, Ayélo AP, Aguemon B, Chouti FL, Zannou MD, et al. (2013) Facteurs de risques des accidents d'exposition au sang chez les professionnels de la santé de la zone sanitaire Parakou-n'dali (nord Bénin). Rev CAMES SANTE 1: 1.

9. Hajjaji Darouiche M, Jmal Hammami K, Gargouri I, Jaziri Boudaya S, Masmoudi ML (2010) Les médecins stagiaires : une population à risque d'accidents d'exposition au sang. À propos d'une étude au CHU de Sfax-Tunisie. Archives des Maladies Professionnelles et de l'Environnement 71: 941-945.

10. Ehui E, Tanon A, Guié P, Aba T, Toa-Lou C, et al. (2010) Prophylaxie antirétrovirale après expositions non professionnelles au VIH à Abidjan (Côte d'Ivoire). Médecine et Maladies Infectieuses 40: 574-581.

11. Ehui E, Kra O, Ouattara I, Eholié S, Kakou A, et al. (2007) Prise en charge des accidents d'exposition au sang au CHU de Treichville, Abidjan (Côte-d'Ivoire). Médecine et Maladies Infectieuses 37: S251-S256.

12. Kara-Pékéti K, Magnang $\mathrm{H}$, Bony J-S, Robin $\mathrm{H}$, Frimat $P$ (2011) Prévalence des accidents professionnels d'exposition au sang chez le personnel soignant au Togo (Afrique). Archives des Maladies Professionnelles et de l'Environnement 72: 363-369.

13. Zoungrana J, Yaméogo TM, Kyelem CG, Aba YT, Sawadogo A, et al. (2014) Blood exposure accidents: Knowledge, attitudes and practices of nursing and midwifery students at the Bobo-Dioulasso teaching hospital (Burkina Faso). Médecine et Santé Tropicales 24: 258-262.

14. Asseray N, Alfandari S, Vandenbussche C, Guery B, Parent $\mathrm{K}$, et al. (1998) Analyse des pratiques et connaissances sur les accidents d'exposition au sang. Médecine et Maladies Infectieuses 28: 612-617.

15. Beghdadli B, Ghomari O, Taleb M, Belhaj Z, Belabed A, et al. (2009) Le personnel à risque d'accidents d'exposition au sang dans un $\mathrm{CHU}$ de l'Ouest algérien. Sante Publique 21: $253-261$

16. OMS (2020) L'OMS publie de nouvelles orientations sur l'autodépistage du VIH à la veille de la Journée mondiale du sida. 